\title{
Nueva fuente de quinoles, la superficie foliar de Pentacalia ledifolia y Pentacalia corymbosa y sus propiedades antifúngicas
}

\author{
Julio A. Pedrozo, Rubén D. Torrenegra*, Alba N. Téllez, Alvaro Granados \\ Departamento de Química, Pontificia Universidad Javeriana, Carrera 7 No. 43-82, Edificio Carlos Ortiz (52), \\ Oficina 201, Bogotá, Colombia
}

\begin{abstract}
RESUMO: "Nova fonte de quinóides, a superficie foliar de Pentacalia lediflora e Pentacalia corymbosa e suas propriedades antifúngicas”. Foi demonstrada a ação antifúngica do extrato clorofórmico e de duas substâncias isoladas da superfície foliar de Pentacalia ledifolia (H.B.K.) Cuatr. e P. corymbosa (Benth) Cuatr. frente aos fungos fitopatógenos Fusarium oxysporum e Botrytis cinerea, cultivados em BDA (batata-dextrose-ágar). Destes extratos foram isolados, além de cumarinas já identificadas em estudos anteriores, dois derivados quinóides: (1-hidroxi-4-oxo-2,5ciclohexadienil) acetato de metila ou jacaranona e (1-hidroxi-4-oxo-2,5-ciclohexadienil) acetato de etila ou metiljacaranona. Para o (1-hidroxi-4-oxo-2,5-ciclohexadienil) acetato de etila foi calculado $\mathrm{CI}_{50}$ de $650 \mu \mathrm{g} / \mathrm{mL}$ para os dois tipos de fungos e o (1-hidroxi-4-oxo-2,5-ciclohexadienil) acetato de metila teve um $\mathrm{CI}_{50}$ de $660 \mu \mathrm{g} / \mathrm{mL}$.
\end{abstract}

Unitermos: Pentacalia ledifolia, Pentacalia corymbosa, Asteraceae, Senecioneae, atividade antifúngica, quinóides.

\begin{abstract}
New source of quinols, the surface of Pentacalia ledifolia and Pentacalia corymbosa leaves and its antifungal activity". Quinols identified in the surface waxes of Pentacalia ledifolia (H.B.K.) Cuatr and $P$. corymbosa (Benth) Cuatr. leaves, possess antifungal activity against Fusarium oxysporum and Botrytis cinerea, cultured on PDA (potato-dextrose-agar) medium. These extracts were prepared by dipping fresh leaves in chloroform for $5 \mathrm{~min}$, and afforded ethyl-(1hydroxy-4-oxocyclohexa-2,5-dien-1-yl) acetate and methyl-(1-hydroxy-4-oxocyclohexa-2,5-dien1 -yl) acetate, the major surface compounds.
\end{abstract}

Keywords: Pentacalia ledifolia, Pentacalia corymbosa, Asteraceae, Senecioneae, antifungical activity, quinols.

\section{INTRODUCCION}

Pentacalia ledifolia (H.B.K.) Cuatr y Pentacalia corymbosa (Benth.) Cuatr. (Asteraceae-Senecioneae), son arbustos autóctonos de los páramos andinos colombianos (altitudes entre 2900-3100 m.s.n.m.), principalmente, de los que circundan a la ciudad de Bogotá; se les utiliza en la medicina tradicional como vulnerarias, antisifilíticas, para curar granos y forúnculos rebeldes, contra úlceras y como remedio muy eficaz para curar la faringitis (GarcíaBarriga, 1975; Pérez-Arbeláez, 1956; Díaz; Cuatrecasas, 1999).

El género Pentacalia ha sido poco estudiado y sólo se reportan en la literatura los estudios en $P$. ephiphytica, $P$. andicola, $P$. firmipes y $P$. archieri, de las cuales se aislaron nerolidol, humuleno, farneseno, curcumeno, santaleno, germacreno D, varios cadinenos, cicloartenol, friedelina y 5-metoxieugenol (Bohlmann; Ziesche, 1979; Bohlmann et al., 1984). En estudios anteriores, de P. corymbosa obtuvimos sitosterol, eicosanol, friedelina, dos cumarinas (escopoletina y 7-geraniloxilescopletina) y dos flavonoides (quercetina y rutina) (Torrenegra et al., 2000).

Derivados quinólicos han sido obtenidos de diversas familias vegetales: Caricaceae (Schwab; Schrerier, 1988), Salicaceae (Julkunen-Tiitto, 1986), Oleaceae (Seya et al., 1989; Kuwajima et al., 1993), Scrophulariaceae (Nicoletti et al., 1988; Abdullahi et al., 1986), Bignoniaceae (Hase et al., 1995), Martyniaceae (Sasaki, 1978), entre otras. El (1-hidroxi-4-oxo-2,5ciclohexandienil) acetato de metilo llamado también jaracanona fue asilado de Jacaranda caucana (Ogura et al., 1976) y de Senecio cannabifolius (Lajide et al., 1996); en esta última especie también se identificó el (1hidroxi-4-oxo-2,5-ciclohexandienil) acetato de etilo o metiljacaranona reportándoseles una acción inhibitoria del crecimiento de la larva de Spodoptera litura, un díptero que ataca plantas de tabaco.

Recientemente nuestro grupo aisló triterpenos de corteza de Bursera graveolens (Burseraceae) y sesquiterpenlactonas de Espeletia killipii (Asteraceae) con actividad citotoxica (Robles et al., 2005; Alfonso et al., 2006; Jaimes et al., 2006). En este trabajo fueron 
aislados quinoles de dos especies de Pentacalia con actividad antimicrobiana.

\section{MATERIAL Y METODOS}

Las plantas Pentacalia corymbosa y P. ledifolia fueron recolectadas en los páramos de "Guasca" y "Cruz Verde", al Norte y al Sureste de la ciudad de Bogotá, respectivamente, a altitudes entre los 2.900-3.100 m.s.n.m.; especímenes de estas plantas fueron radicados en el Herbario Nacional de Colombia bajo los número COL407570 y COL404447.

Unos $200 \mathrm{~g}$ de hojas frescas de ambas plantas fueron lavadas por inmersión en $\mathrm{CHCl}_{3}(5 \mathrm{~mL} / \mathrm{g}$ material vegetal), durante cinco minutos; los extractos resultantes fueron concentrados a mínimo volumen en un evaporador rotatorio; el concentrado resultante fue tratado con $\mathrm{Me}_{2} \mathrm{CO}$ y dejado en reposo a $4{ }^{\circ} \mathrm{C}$ durante 12 horas. El flóculo producido (constituido casi exclusivamente por ceras y grasas) fue removido por filtración al vacío y el filtrado resultante, se concentró a presión reducida en el evaporador rotatorio y se secó al ambiente. Los porcentajes de rendimiento ( $\mathrm{g}$ de extracto/100 g de material fresco) fueron de 0,9 para Pentacalia corymbosa y de 0,8 para $P$. ledifolia. Estos extractos fueron ensayados ante las dos cepas fúngicas.

Tres gramos (3 g) de dicho extracto se percolaron a través de columna de sílica gel (Kieselgel, Merck 0,2-0,060 mm) la cual se eluyó con bencina de petróleo y mezclas de bencina de petróleo-acetato de etilo de polaridad creciente. Las fracciones recolectadas fueron monitoreadas con cromatografías en capa delgada (CCD) y, las sustancias aisladas fueron purificadas por cromatografías en capa delgada preparativas (CCDP).

Los espectros de RMN fueron obtenidos en un espectrómetro Bruker AMX400 a $400 \mathrm{MHz}$ para los de ${ }^{1} \mathrm{H}$ y a $100 \mathrm{MHz}$ para los de ${ }^{13} \mathrm{C}$, con TMS como estándar interno; los espectros IR fueron tomados como pastillas de $\mathrm{KBr}$, en un instrumento Shimadzu, modelo 8300 Series FTIR y, los espectros de masas por impacto electrónico (EIMS) fueron obtenidos en un espectrómetro AEIMS 902 de doble haz, usando una sonda directa operando a $70 \mathrm{eV}(97 \mathrm{~kJ} / \mathrm{mol})$ y $110-160^{\circ}$.

1-Hidroxi-4-oxo-2,5-ciclohexandienil acetato de metilo o jacaranona (P-1). Cristalizó en forma de agujas incoloras con $\mathrm{Pf}=72{ }^{\circ} \mathrm{C}$, a partir de $\mathrm{CHCl}_{3}$. Rf 0,4 en placas de sílica corridas con $\mathrm{CHCl}_{3}-\mathrm{MeCO}$ 9:1; absorbió la flurescencia de las placas de sílica a la luz UV de onda corta y, reveló amarillo ocre con vainillina/ $\mathrm{H}_{2} \mathrm{SO}_{4}$. IR, en pastilla de $\mathrm{KBr}: v$ en $\mathrm{cm}^{-1}$ (intensidad, clase de hidrógeno) 3.265 (I, OH); 2.956 (I, CH); 1.735 (M, $\mathrm{CO}$ de ésteres); $1.666(\mathrm{M}$, cetona $\alpha, \beta$-insaturadas cíclicas de seis miembros); $1.612(\mathrm{M}, \mathrm{C}=\mathrm{C}) ; 1.247(\mathrm{D}, \mathrm{CO}$ para ésteres), 1.072 (M, C-O de alcohol). RMN ${ }^{1} \mathrm{H}$ (400 MHz, en $\mathrm{CDCl}_{3}$ ): $\delta$ en ppm (señal, número de hidrógenos, clase): 6,17 (dd, $2 \mathrm{H},-\mathrm{CH}=\mathrm{CH}-\mathrm{COH}-$ ) $\mathrm{J}=10,2 \mathrm{~Hz}$ y J=6,5 $\mathrm{Hz} ; 6,94$ (dd, 2H, -CH=Cㅍ-CO-) $\mathrm{J}=10,2 \mathrm{~Hz}$ y $\mathrm{J}=6,5$
$\mathrm{Hz} ; 2,69$ (s, 2H, - $\left.\underline{\mathrm{H}}_{2}-\mathrm{CO}-\right)$ y 3,74 (s, 3H, -CO-OC $\left.\underline{\mathrm{H}}_{3}\right)$. $\mathrm{RMN}^{13} \mathrm{C}\left(\mathrm{J}-\mathrm{mod}\right.$, en $\left.\mathrm{CDCl}_{3}\right): \delta$ en ppm (clase de carbono) 52,44 (-CO-OCH$\left.{ }_{3}\right) ; 43,61$ (- $\left.\underline{\mathrm{CH}}_{2}-\mathrm{CO}-\right) ; 67,49$ (>C $\left.-\mathrm{CH}\right)$;

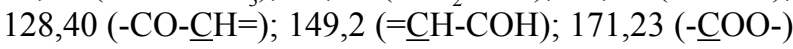
y 185,17 (- $\underline{\mathrm{CO}}-\mathrm{CH}=)$. EIMS: m/z (intensidad en \%) 182 $\mathrm{M}^{+}(8), 169$ (5), 150 (18), 122 (17), 109 (100), 94 (7), 81 (39), 74 (64), 69 (15), 43 (38).

1-Hidroxi-4-oxo-2,5-ciclohexandienil acetato de etilo o metiljacaranona (P-2). Cristalizó en forma de agujas incoloras con $\mathrm{Pf}=54{ }^{\circ} \mathrm{C}$, a partir de $\mathrm{CHCl}_{3}$. Rf 0,33 en placas de sílica corridas con $\mathrm{CHCl}_{3}-\mathrm{MeCO}$ 9:1; absorbió la flurescencia de las placas de sílica a la luz UV de onda corta y reveló amarillo ocre con vainillina/ $\mathrm{H}_{2} \mathrm{SO}_{4}$. IR, en pastilla de $\mathrm{KBr}: v$ en $\mathrm{cm}^{-1}$ (intensidad, tipo de enlace) $3.266(\mathrm{I}, \mathrm{OH}) ; 2.958$ (I, CH); 1.725 (M, CO de ésteres); $1.664(\mathrm{M}$, cetona $\alpha, \beta$-insaturadas cíclicas de seis miembros); $1.616(\mathrm{M}, \mathrm{C}=\mathrm{C}) ; 1.242(\mathrm{D}, \mathrm{CO}$ para ésteres), 1.068 (M, C-O de alcohol). RMN ${ }^{1} \mathrm{H}$ (400 MHz, en $\mathrm{CDCl}_{3}$ ): $\delta$ en ppm (señal, número de hidrógenos, clase) 1,29 (t, 3H, $\left.-\mathrm{CH}_{2}-\mathrm{CH}_{3}\right) \mathrm{J}=7 \mathrm{~Hz} ; 2,69$ (s, 2H, >C$\mathrm{CH}_{2}-\mathrm{COO}-$ ); 4,22 (q, 2H, -CO- $\mathrm{CH}_{2}-\mathrm{CH}_{3}$ ) J=7 Hz; 6,19 $(\mathrm{dd}, 2 \mathrm{H},-\mathrm{C} \underline{\mathrm{H}}=\mathrm{CH}-) \mathrm{J}=10,2 \mathrm{~Hz}$ y $\mathrm{J}=6,5 \mathrm{~Hz} ; 6,96$ (dd, 2H, $-\mathrm{C} \underline{\mathrm{H}}=\mathrm{CH}-) \mathrm{J}=10,2 \mathrm{~Hz}$ y $\mathrm{J}=6,5 \mathrm{~Hz} . \mathrm{RMN}^{13} \mathrm{C}(\mathrm{J}-\mathrm{mod}$, en $\left.\mathrm{CDCl}_{3}\right): \delta$ en ppm (tipo de carbono) 14,28 $\left(\mathrm{CH}_{3}-\right) ; 43,69$ $\left(-\underline{\mathrm{CH}}_{2}-\mathrm{COO}\right) ; 61,73\left(\mathrm{CO}-\underline{\mathrm{CH}}_{2}-\right) ; 128,49$ (-CO- $\left.\underline{\mathrm{CH}}=\mathrm{CH}-\right)$; $149,13(-\mathrm{CH}=\underline{\mathrm{C}} \mathrm{H}-\mathrm{C}-\mathrm{OH}) ; 171,11$ (ㅁO) y 185,15 (-ㅁ$\mathrm{CH}=)$.

\section{RESULTADOS Y DISCUSION}

A los extractos de las sustancias de la superficie foliar de Pentacalia corymbosa (1h-Pc) y P. ledifolia (1h$\mathrm{Pl})$, se les evaluó la acción inhibitoria del crecimiento hifal de Fusarium oxysporum f. sp. dianthi y Botrytis cinerea, responsables de infecciones en plantas de claveles y rosas cultivadas en invernaderos. Para ello se utilizó el método de crecimiento radial en medio PDA (papa-dextrosa-agar) $\mathrm{y}$, la griseofulvina (GRSF) como control positivo (Paxton, 1991; Torrenegra et al., 2000). Estos extractos mostraron concentraciones inhibitorias medias $\left(\mathrm{CI}_{50}\right)$ entre 1,8 y 2,0 $\mathrm{mg} / \mathrm{mL}$, como se puede observar en la tabla 1 .

De los extractos $1 \mathrm{~h}-\mathrm{Pc}$ y $1 \mathrm{~h}-\mathrm{Pl}$ se aislaron eicosanol, ácido eicosanoico, acetato de $\beta$-amirina y dos cumarinas: la escopoletina y el 7-geraniloxilescopoletina, ya reportados en estudios anteriores (Torrenegra et al., 2000). Además, se obtuvieron dos sólidos que se rotularon como P-1 y P-2.

El sólido P-1 se presentó como un aceite coloreado que cristalizó lentamente, a partir de $\mathrm{CHCl}_{3}$, en formas de agujas incoloras. El espectro IR mostró señales para $-\mathrm{OH}$ $\left(3.265 \mathrm{~cm}^{-1}\right)$, para $\mathrm{CO}$ de éster $\left(1.735 \mathrm{~cm}^{-1}\right)$, para $\mathrm{CO}$ de cetona $\alpha, \beta$-insaturada $\left(1.666 \mathrm{~cm}^{-1}\right)$ y para insaturaciones $\mathrm{C}=\mathrm{C}\left(1.612 \mathrm{~cm}^{-1}\right)$. El espectro $\mathrm{RMN}^{1} \mathrm{H}$ sólo presentó 4 señales: a $\delta 2,69$ de un singulete de metileno ligado a carbono carbonilo; a $\delta 3,74$ de un singulete de oximetilo de un éster y, dos a $\delta 6,17$ (dd) y $\delta 6,94$ (dd) de hidrógenos vinílicos con acoplamientos orto y meta $(\mathrm{J}=10,2 \mathrm{~Hz}$ y 
Tabla 1. Concentraciones inhibitorias medias $\left(\mathrm{CI}_{50}\right)$ en $\mathrm{mg} / \mathrm{mL}$ de los extractos de las sustancias de las superficies foliares de Pentacalia corymbosa (1h-Pc) y P. ledifolia (1h-Pl).

\begin{tabular}{ccc}
\hline Extractos vegetales & \multicolumn{3}{c}{ Cepas fúngicas } \\
\cline { 2 - 3 } & F. oxysporum & B. cinerea \\
\hline $1 \mathrm{~h}-\mathrm{Pc}$ & $1,8 \mathrm{mg} / \mathrm{mL}$ & $1,9 \mathrm{mg} / \mathrm{mL}$ \\
$1 \mathrm{~h}-\mathrm{Pl}$ & $1,9 \mathrm{mg} / \mathrm{mL}$ & $2,0 \mathrm{mg} / \mathrm{mL}$ \\
\hline
\end{tabular}

Tabla 2. Inhibición en porcentajes (\%I) para concentraciones entre 125-750 $\mu \mathrm{g} / \mathrm{mL}$ de (1-hidroxi-4-oxo-2,5ciclohexandienil) acetato de metilo o jacaranona.

\begin{tabular}{lllll}
\hline \multicolumn{1}{c}{ Concentración $\mu \mathrm{g} / \mathrm{mL}$} & 750 & 500 & 250 & 125 \\
\hline Fusarium oxysporum & 57,0 & 37,8 & 13,5 & 5,2 \\
Botrytis cinerea & 55,5 & 37,0 & 12,9 & 5,1
\end{tabular}

Tabla 3. Inhibición en porcentajes (\%I) para concentraciones entre 125-750 $\mu \mathrm{g} / \mathrm{mL}$ de (1-hidroxi-4-oxo-2,5ciclohexandienil) acetato de etilo o metiljacaranona.

\begin{tabular}{lcccc}
\hline \multicolumn{1}{c}{ Concentraciones en $\mu \mathrm{g} / \mathrm{mL}$} & 750 & 500 & 250 & 125 \\
\hline Fusarium oxysporum & 59,3 & 38,4 & 21,0 & 10,4 \\
Botrytis cinerea & 58,6 & 37,9 & 20,4 & 9,8 \\
\hline
\end{tabular}<smiles>[1H]C(=O)OCC(C)=O</smiles>

Figura 1. Asignaciones a las señales del espectro $\mathrm{RMN}^{1} \mathrm{H}$ de P-1 (1-hidroxi-4-oxo-2,5-ciclohexandienil) acetato de metilo o jacaranona.<smiles>CCOC(=O)CC1(C)C=CC(=O)C=C1</smiles>

Figura 2. Asignaciones de las señales del espectro $\mathrm{RMN}^{1} \mathrm{H}$ de P-2 (1-hidroxi-4-oxo-2,5-ciclohexandienil) acetato de etilo o metiljacaranona. 
6,32 Hz), de un sistema AA'BB'. En el espectro $\mathrm{RMN}^{13} \mathrm{C}$ aparecieron 7 señales, a $\delta$ 43,63 de un metileno unido a carbono carbonilo; a $\delta 52,44$ para $\underline{\mathrm{CH}}_{3} \mathrm{O}-\mathrm{CO}$; dos (cada una correspondiente a dos carbonos) para $>\underline{\mathrm{C}} \mathrm{H}-(\delta 128,40$ y 149,20) y tres para carbonos cuaternarios; uno de los carbonos cuaternarios es un carbonilo de éster $(\delta 171,13)$, otro es un carbonilo de cetona cíclica $(\delta 185,17)$ y el último posee un grupo hidroxilo $(\delta 67,49)$. En el espectro EIMS se observó un pico progenitor $\mathrm{M}^{+}$con $\mathrm{m} / \mathrm{z} 182$, lo que permitió asignarle a este compuesto una fórmula molecular $\mathrm{C}_{9} \mathrm{H}_{10} \mathrm{O}_{4}$. Las propiedades espectroscópicas y el punto de fusión (72 $\left.{ }^{\circ} \mathrm{C}\right)$, coincidieron con los reportados en la literatura para la jacaranona o (1-hidroxi-4-oxo-2,5-ciclohexandienil) acetato de metilo (Ogura et al., 1976; Lajide et al., 1996).

El sólido P-2 no presentó en su espectro $\mathrm{RMN}^{1} \mathrm{H}$ la señal a $\delta 3,79$ del grupo oximetilo de éster presente en el espectro del sólido P-1, pero en cambio aparecieron dos señales más: una (q) a $\delta$ 4,22 para un oximetileno de éster y otra $(\mathrm{t})$ a $\delta 1,29$ de un metilo, mutuamente acoplados $(\mathrm{J}=7 \mathrm{~Hz})$. En el espectro $\mathrm{RMN}^{13} \mathrm{C}$ aparecieron también dos señales diferentes: una a $\delta 61,73$ para un oximetileno de éster y otra a $\delta 14,28$ para un metilo unido a metileno. El análisis permitió asignar a este sólido una fórmula molecular $\mathrm{C}_{10} \mathrm{H}_{12} \mathrm{O}_{4}$; su punto de fusión ( $\mathrm{Pf}=$ $54{ }^{\circ} \mathrm{C}$ ) coincidió con el reportado en la literatura para el metiljacaranona o (1-hidroxi-4-oxo-2,5-ciclohexandienil) acetato de etilo (Lajide et al., 1996).

Para los quinoles aislados se prepararon soluciones de $30,20,10$ y $5 \mathrm{mg} / \mathrm{mL}$, de las cuales se tomaron $150 \mu \mathrm{L}$ para adicionarlos a $6 \mathrm{~mL}$ de medio de cultivo PDA contenido en cajas de Petri obteniéndose concentraciones de 750, 500, 250 y $125 \mu \mathrm{g} / \mathrm{mL}$ respectivamente. Las cajas se inocularon con Fusarium oxysporun f. sp. dianthi y Botrytis cinerea y se incubaron durante 6 días a $25^{\circ} \mathrm{C}$; al término del tiempo se midieron los diámetros de las colonias y se calcularon los \% $\mathrm{C}$ y $\%$ I. En las tablas 2 y 3 se ordenan los valores de \%I y sus respectivas concentraciones, para los dos quinoles.

Se graficaron los valores de \%I contra sus respetivas concentraciones y se determinaron, por interpolación, los valores aproximados de $\mathrm{CI}_{50}$ para los dos quinoles; para el (1-hidroxi-4-oxo-2,5-ciclohandienil) acetato de metilo se encontró una $\mathrm{CI}_{50}$ de $660 \mu \mathrm{g} / \mathrm{mL}$ y para el (1-hidroxi-4-oxo- 2,5-ciclohexandienil) acetato de etilo una de $650 \mu \mathrm{g} / \mathrm{mL}$.

Al comparar la baja acción antifúngica de los quinoles frente a la obtenida para cumarinas (Torrenegra et al., 2000), se podría sugerir que la presencia de estos compuestos en la superficie foliar sea la de un papel defensivo contra herbívoros (Lajide et al., 1996) y no contra hongos.

\section{AGRADECIMIENTOS}

Los autores agradecen al doctor Alexander Gray de la Universidad de Strathclyde (Escocia) por los espectros de $\mathrm{RMN}^{1} \mathrm{H}$ y $\mathrm{RMN}^{13} \mathrm{C}$; a la Universidad

Javeriana y a COLCIENCIAS por su apoyo económico (Proyecto código 1203-05-394-95,CT-128-97).

\section{REFERENCIAS}

Abdullahi H, Nyandat E, Galeffi C, Mesana I, Nicoletti M, Marini-Bettolo G 1986. Cyclohexanols of Halleria lucida. Phytochemistry 25: 2821-2823.

Alfonso ANT, Castro C, Murcia TR, Torrenegra R 2006 Efectos citotoxicos in vitro de extractos y fracciones de Espeletia killipii Cuatr. frente a lineas celulares tumorales humanos. Rev Bras Farmacogn 16: 12-16.

Bohlmann F, Ziesche J 1979. Neue germacrene-derivateaus Senecio-arten. Phytochemistry 18: 1489-1494.

Bohlmann F, Castro V, Ziesche J 1984. New sesquiterpenes from Pentacalia species. Rev Latinoamer Quím 14: 103-106.

Díaz S, Cuatrecasas J 1999. Asteraceas de la flora de Colombia Senecioneae-1. Gérneros Deridrophorbium y Pentacalia. Academia Colombiana de Ciencias Exactas, Físicas y Naturales. Colección Jorge Alvarez Lleras No. 12. Bogotá, Colombia. 345 p.

García-Barriga H 1975. Flora medicinal de Colombia. Vol. 2. Editorial Imprenta Nacional. Bogotá, Colombia. pg. 293, 398-409.

Hase T, Kawamoto Y, Ohtani K, Kasai R, Yamasaki K, Picheamsoonthon CH 1995. Cyclohexylethanoids and related glucoside from Millingtonia hortensis. Phytochemistry 39:235-241.

Jaimes G, Castro C, Aristizaba FA, Murcia TR, Torrenegra R, Alfonso ANT 2006. Principio activo citotoxico de Espeletia killipii Cuatr. sobre células tumorales y su toxicidad frente a células normales humanas. Rev Bras Farmacogn 16: 140-145.

Julkunen-Tiitto R 1986. A chemotaxonomic survey of phenolics in leaves of Northen Salicaceae species. Phytochemistry 25:663-667.

Kuwajima $\mathrm{H}$, Takahashi $\mathrm{M}$, Ito $\mathrm{M}, \mathrm{Wu} \mathrm{H}$, Takaishi $\mathrm{K}$, Inoue K 1993. A quinol glucoside from Abeliophyllum distichum. Phytochemistry 33:137-139.

Lajide L, Escoubas P, Mizutani J 1996. Cyclohexadienonesinsect growth inhibitors from the foliar surface and tissue extracts of Senecio cannabifolius. Experientia 52: 259-263.

Nicoletti M, Galeffi C, Messana I, Marini-Betolo G, Garbarino J, Gambaro V 1988. Phenylpropanoid glycosides from Calceolaria hypericina. Phytochemistry 27: 639-641.

Ogura M, Cordell G, Farnsworth N 1976. Potencial anticancer agents. III. Jacaranone, a novel phytoquinoid from Jacaranda caucana. Lloydia 39: 255-257.

Paxton J 1991. Assays for Antifungal Activity. In: Methods in Plant Biochemistry. Vol. 6. Assays for Bioactivity. Hostettmann, K. (Ed). Academic Press. London, England. pp. 33-46.

Pérez-Arbeláe E 1956. Plantas útiles de Colombia. Librería colombiana, Camacho Roldán Ltda. Bogotá, Colombia. pg. 229-230.

Robles J, Torrenegra R, Gray AI, Piñeros C, Ortiz L, Sierra M 2005. Triterpenos aislados de corteza de Bursera graveolens (Burseraceae) y su actividad biologica. Rev Bras Farmacogn 15: 283-286.

Sasaki H, Taguchi H, Endo T, Yosioka I, Higashiyama K, 
Otomasu H 1978. The glycosides of Martynia louisiana Mill, a new phenylpropanoid glycoside, Martynoside. Chem Pharm Bull 26: 2111-2121.

Schwab W, Schreier P 1988. Aryl- $\beta$-D-glucosides from Carica papaya fruit. Phytochemistry 27: 1813-1816.

Seya K, Endo K, Hikino H 1989. Structures of rengyosides A, B and $\mathrm{C}$, three glucosides of Forsythia suspensa fruits. Phytochemistry 28: 1495-1498.

Torrenegra RD, Pedrozo JA, Téllez AN, Cabeza G, Granados A, Méndez D 2000. Química y actividad antifúngica de Pentacalia corymbosa (Asteraceae-Senecioneae). Rev Latinoamer Quím 28: 31-34. 